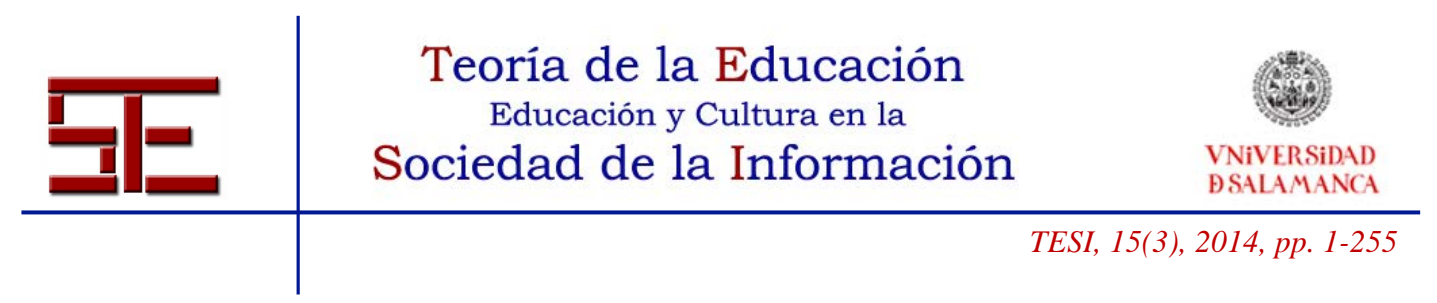

\title{
MODELO DE ADMINISTRACIÓN DEL CONOCIMIENTO EN SISTEMAS MÓVILES APLICADOS A LA CAPACITACIÓN
}

Chadwick CARRETO

ARELLANO

ESCOM-IPN

ccarretoa@ipn.mx

Eduardo BUSTOS

FARIAS

ESCOM-IPN

ebustosf@ipn.mx
Elena Fabiola RUIZ

LEDESMA

ESCOM-IPN

elen_fruiz@yahoo.com.mx
Saida Nelly SUÁREZ

BETANCOURT

ESCOM-IPN

flais2213@hotmail.com

Resumen:

Mariana VICARIO

SOLÓRZANO

UPIICSA-IPN

cvicario@ipn.mx

En el presente trabajo se muestra el desarrollo de un Modelo de Administración del Conocimiento (MAC) aplicado al proceso de Capacitación para personal en dispositivos móviles para facilitar el uso y acceso de diferentes tipos de usuarios a información pertinente en cualquier lugar y a cualquier hora (anytime, anywhere). El MAC permite administrar el conocimiento, de tal forma que ayude en el proceso de recopilación, clasificación y búsqueda de información de acuerdo a un perfil y necesidades específicas de capacitación, además de servicios asociados a la transformación de datos e información para la generación de conocimiento. El MAC pretende poner a disposición de los usuarios, herramientas para el desarrollo de competencias y permitir desarrollar el proceso de capacitación con el uso de dispositivo de capacidad limitada con conexión a Internet.

Palabras clave: Administración del Conocimiento, Proceso de Capacitación, Dispositivo Móvil. 


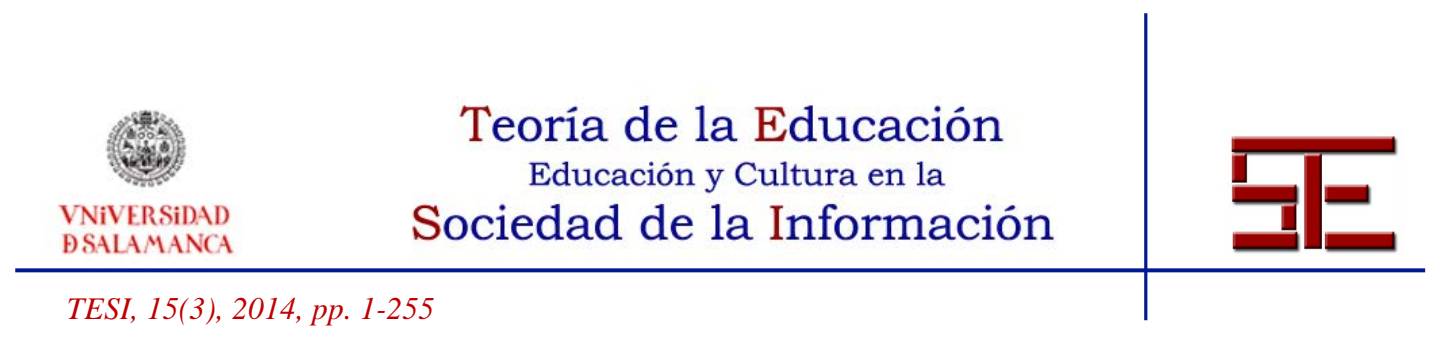

MODEL OF KNOWLEDGE MANAGEMENT IN MOBILE SYSTEMS USED FOR TRAINING

\begin{abstract}
:
This work shows the development of a Knowledge Management Model (MAC) applied to the training process in mobile devices for ease of use and access of different types of users to relevant information (anywhere and anytime). The MAC permit to manage knowledge, so that helps in the process of collection, classification and search of information according to a profile and academic needs as well as services related to the transformation of data and information for knowledge generation. The MAC aims to provide users, tools for skills development and allow the development of the training process with the use of limited capacity device with Internet connection.
\end{abstract}

Key words: Knowledge Management, Teaching-Learning Process, Mobile Devices. 


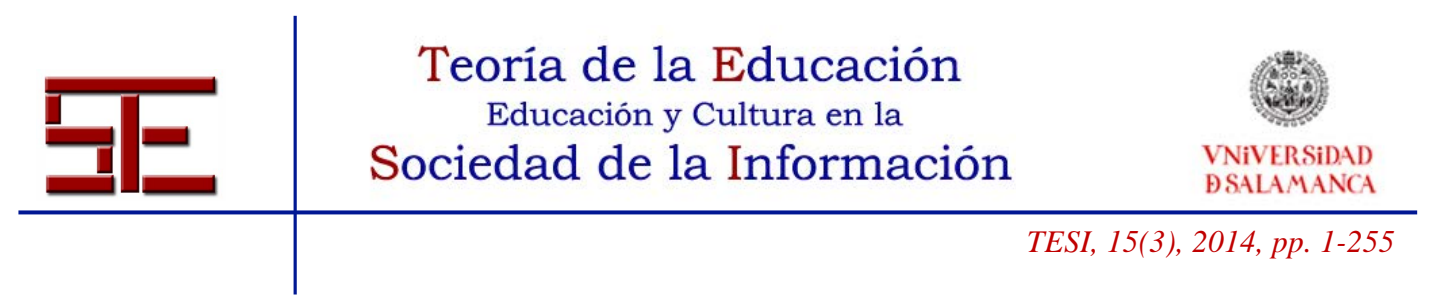

\section{INTRODUCCIÓN}

El conocimiento, cualquiera que sea su tipo o procedencia, es un activo de naturaleza intangible y, en consecuencia, invisible y de difícil valoración. Entre las características del ser humano, se encuentra la capacidad para aprovechar sus propias experiencias y convertirlas en acciones susceptibles de ser generalizadas para su transmisión a las generaciones subsecuentes, dicha cualidad natural del individuo se ha venido transformando en un proceso sistemático y paulatino de conocimientos, cuyo propósito ha sido, entre otros, una mayor accesibilidad al desarrollo personal, para incrementar, en su caso, la capacidad de adaptación del ser humano a las demandas de su entorno y su contexto histórico.

La capacitación en el trabajo no puede escapar al concepto de la educación continua, que pueda significar un verdadero proceso de aprendizaje y un cambio de actitudes del individuo, en beneficio de una mayor y mejor capacidad de conocimiento, ya que quien aprende es un agente que genera su propia información, para ratificar o rectificar sus acciones, sus hábitos y comprender en forma más real su ubicación en el contexto social en el que se encuentra inmerso.

La capacitación del personal de cualquier empresa es uno de los desafíos más importantes que enfrentan las empresas y organizaciones, pues existe la necesidad constante de que estas se adapten al cambio, así mismo se supone que puede generar potencialmente mayor productividad. La globalización de la economía ha creado muchas oportunidades para las empresas y organizaciones, pero también ha creado un sin número de retos, especialmente para las empresas de este tipo que desean tener una presencia global, o que envían a sus trabajadores a asignaciones en el extranjero, para poder comunicarse y colaborar.

El factor humano es la variable más importante de cualquier organización, sin que por ello se desmerite la importancia de los recursos materiales, tecnológicos o financieros, sin embargo las personas representan el activo más importante de la empresa, por aspectos tales como su capacidad, su vulnerabilidad, su maleabilidad o bien sus conocimientos, considerados como uno de los factores que mayores beneficios o perjuicios.

Es por esta situación que se requiere de personas mejor capacitadas, comunicadas y organizadas para enfrentar los cambios que demandan las organizaciones, cambios con un enfoque de desarrollo integral, considerando que los modelos tradicionales y actuales de la administración se encuentran en una etapa crítica, al no resolver los problemas y cubrir o satisfacer las necesidades que demanda la sociedad actual es necesario migrar a las soluciones de Administración del Conocimiento y generación de transferencia de conocimiento.

La gestión del conocimiento (Knowledge Management) posee la capacidad de regenerar

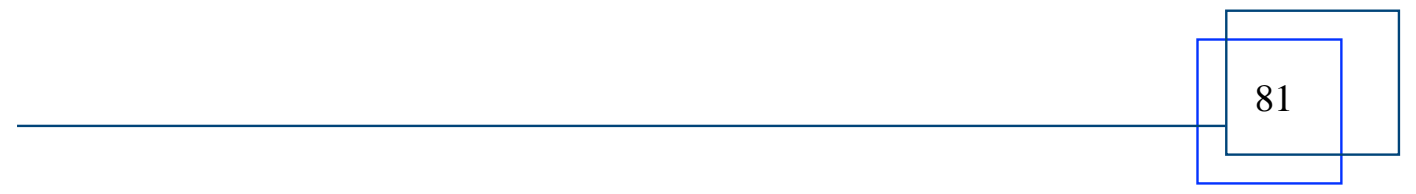




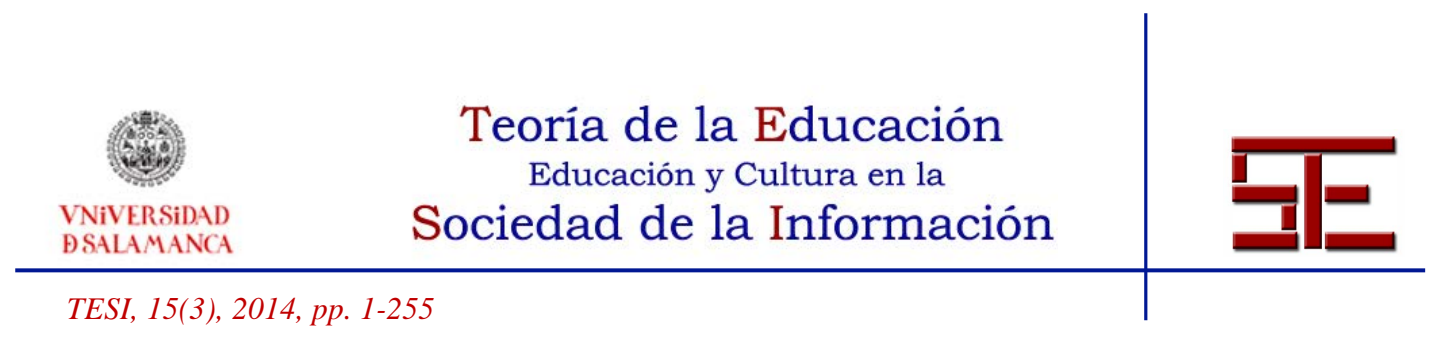

el conocimiento y provocar el aprendizaje. (Dutta y De Meyer, 1997) lo definen como la habilidad de las personas para entender y manejar la información utilizando la tecnología y la compartición de conocimiento.

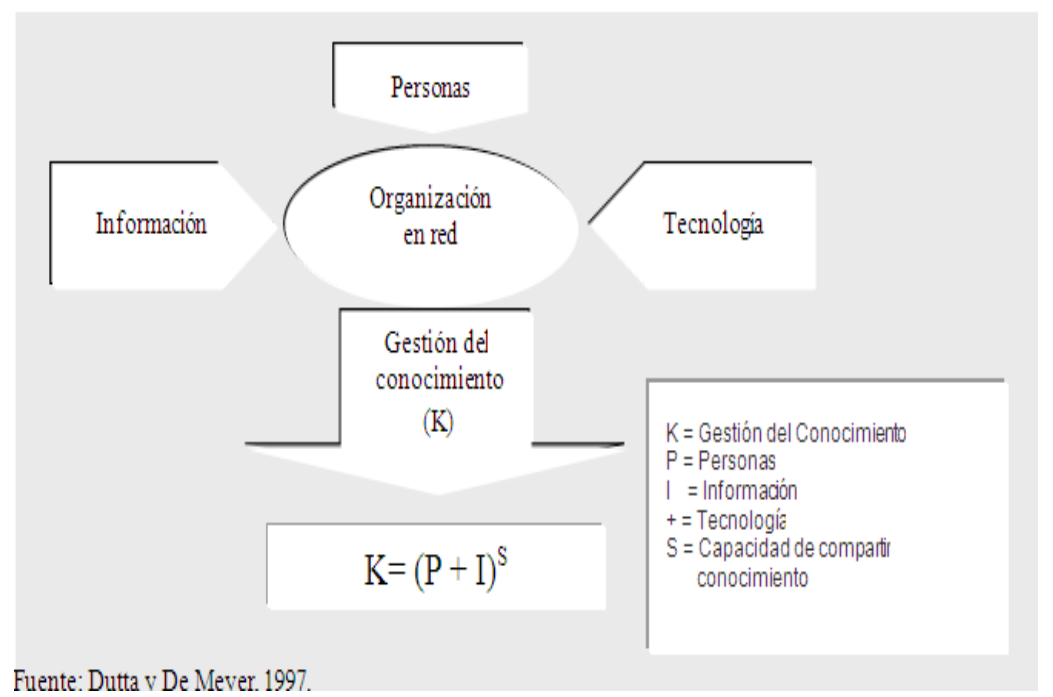

Figura 1. Gestión del Conocimiento.

Sin embargo, la mayoría de los autores especialistas en el tema acuerdan definir la gestión del conocimiento apoyándose en las diferentes etapas de las que se compone y/o en sus objetivos (Vygotsky, 2008).

De acuerdo a la problemática antes citada, es importante que las empresas definan modelos de gestión de conocimiento que permitan a su Capital Humano el desarrollo de su capacidades personales por medio de esquemas que permitan su capacitación y generación de conocimiento de forma práctica, sencilla y económica.

En la sección II se describirá el Modelo de Administración de Conocimiento, posteriormente en la sección III se analiza y se diseña el modelo propuesto a continuación en la sección IV se describirá la implementación del modelo y finalmente en la sección V se presentaran las conclusiones y el trabajo a futuro.

\section{MODELO DE ADMINISTRACIÓN DE CONOCIMIENTO (MAC)}

Sabemos que en la mayoría de las organizaciones la necesidad de información y acceso a ella son completamente distintas, la administración de toda la información es casi nula y es sumamente difícil tener la información que requerimos en el lugar y el momento oportuno. Además el tener toda la información al alcance de todos los usuarios siempre es un grave riesgo, hablando de seguridad y otros aspectos. Lo que se pretende es una

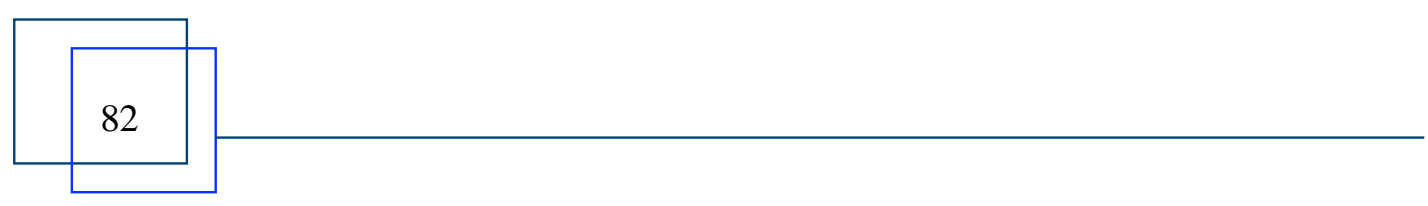




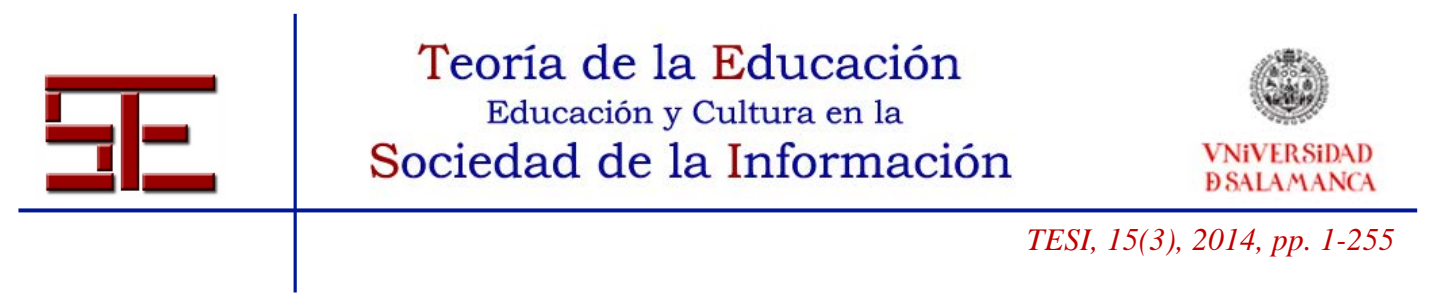

reestructuración en el acceso y administración de tal información por medio de administración del conocimiento, teniéndose el acceso a la información que sea de utilidad y que permita un conocimiento significativo (Prusak, 2001).

Como se comentó anteriormente, un MAC está conformado por varias etapas de depuración y que buscan convertir los datos en información y está en conocimiento que resulte útil de acuerdo a los perfiles y necesidades de los usuarios. Estas etapas son:

- Identificar, recoger y organizar datos e información de acuerdo a perfiles.

- Identificar y obtener el conocimiento existente.

- Depurar los datos e información para facilitar la creación del nuevo conocimiento.

- Iniciar la innovación a través de la reutilización y apoyo de la habilidad del conocimiento generado, por medio de herramientas de acceso a este.

- Aplicar los conocimientos facilitando el proceso de enseñanza.

Dentro de este modelo la etapa de depurar los datos y convertirlos en el llamado "Entendimiento" es de vital importancia, pues esto permitirá aplicar todo este conocimiento en un proceso de enseñanza.

La enseñanza es un campo en el que bien se podría aplicar la tecnología inalámbrica y donde es necesario administrar los resultados obtenidos, ya que permite establecer un ambiente de generación de conocimiento que no se reduce a un espacio físico limitado bajo ciertos requerimientos de conexión; incluso el salón se podría convertir en laboratorio o la cafetería en biblioteca. Esta tecnología proporciona cambios fundamentales en la forma en que se difunden las ideas, el conocimiento y la comprensión (Muñoz Duarte, 2003). Los Modelos de aprendizaje pueden generar grandes ventajas educativas si se manejan adecuadamente. Una visión general de este modelo lo podemos apreciar en la figura 2:

En las secciones siguientes se plantea la problemática encontrada en la implementación de dispositivos móviles en ámbitos de Enseñanza y la propuesta generada para el diseño y desarrollo de un Modelo de Administración del Conocimiento que permita lo antes mencionado.

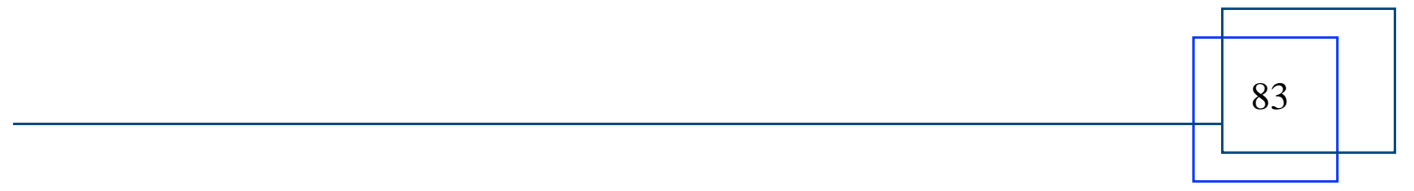



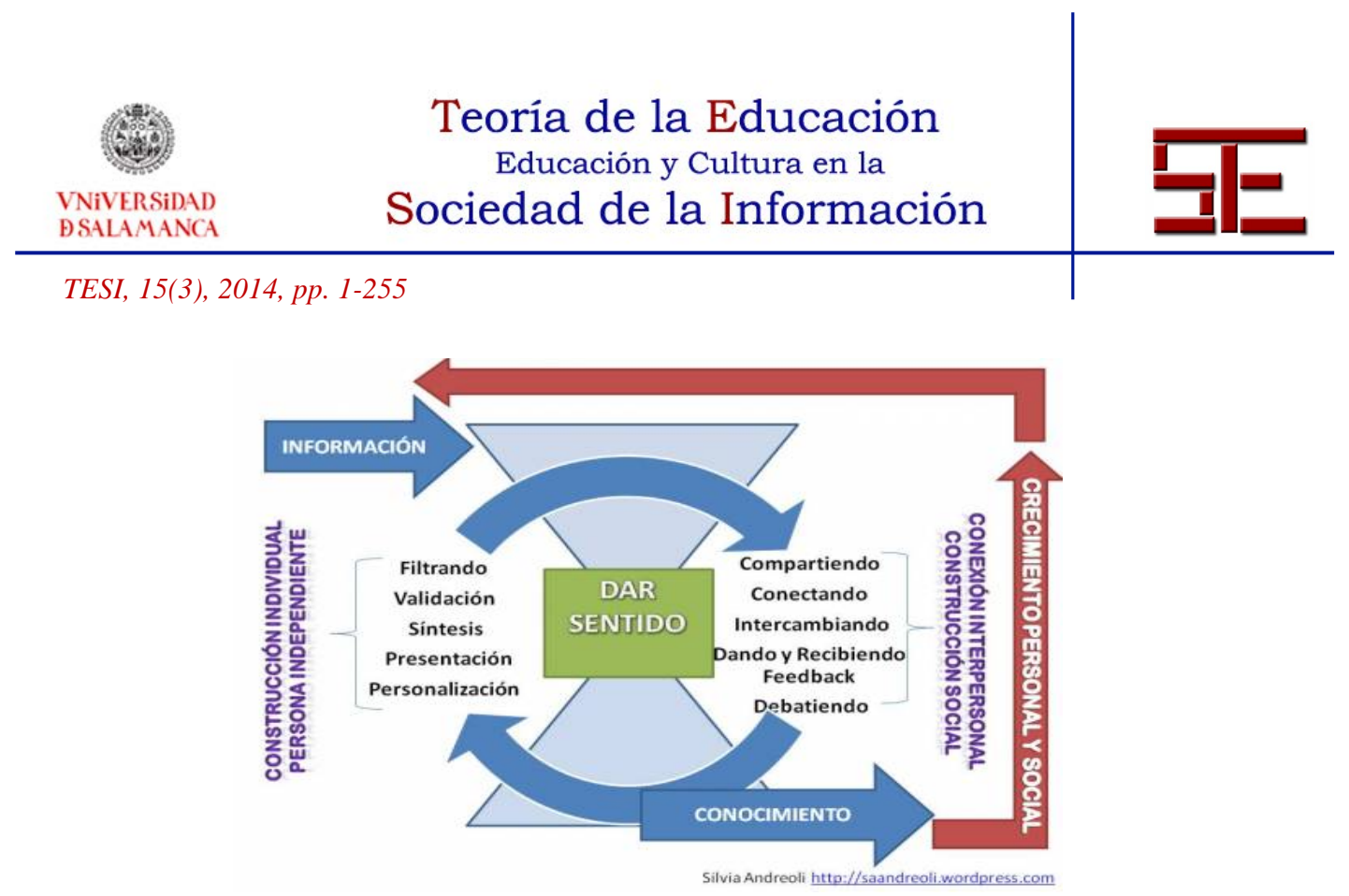

Figura 2. Gestión del Conocimiento.

\section{DISEÑO Y DESARROLLO DEL MODELO DE ADMINISTRACIÓN DEL CONOCIMIENTO}

Uno de los problemas en la educación que existe es una desconexión entre lo que se aprende en el salón de clases y el mundo exterior. Los estudiantes tienen dificultad para apropiarse de los conceptos o aplicar el conocimiento adquirido en las aulas en otros contextos diferentes.

Para atenuar este problema, se propone proporcionar al estudiante herramientas que amplíen y extiendan sus procesos cognitivos, todo esto basado en el Modelo de Administración del Conocimiento propuesto anteriormente.

Los estudiantes pueden aprenden mejor cuando se les da la oportunidad de formarse en las habilidades y teorías dentro del contexto en el que se usan; ellos pueden construir entonces su interpretación personal del tema y comunicar esta interpretación a otros. Dicho de otra forma, es sumergir al estudiante en los problemas de la vida real y promover la investigación, colaboración y creación de competencias para solucionar un problema.

Los dispositivos computacionales móviles pueden apoyar estos procesos ayudando a los estudiantes a buscar y encontrar información dependiendo del contexto, construir su entendimiento y compartirlo con otros. Tal proceso involucra proveer información apropiada al contexto o apoyar a completar una tarea específica en el momento más apropiado. Esta es una forma de tender un puente tecnológico entre la escuela y el lugar de trabajo. Así, el aprendizaje móvil nos da la opción de poder aprovechar la generación de conocimiento.

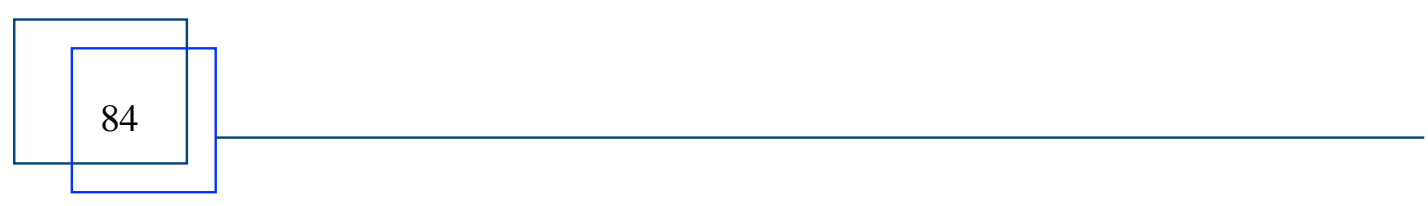




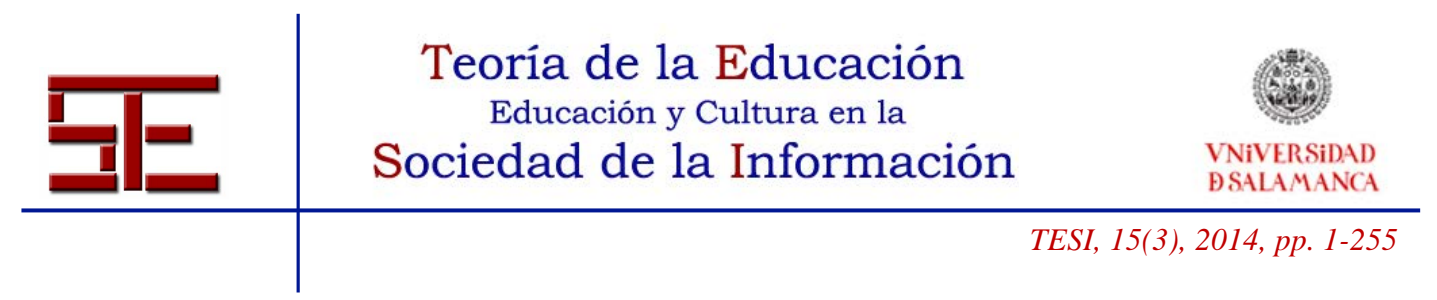

El principal objetivo del aprendizaje móvil es apoyar el "aprendizaje en cualquier momento, en cualquier lugar", lo que no excluye al salón de clases, donde el enfoque es proporcionar apoyo para que los estudiantes manejen conceptos complejos explorando datos e ideas, tengan más autonomía y colaboren de forma más efectiva. Deben existir herramienta de administración, configuración, servicios, conectividad, autentificación, localización de dominios e identificación de dispositivos (Suárez, Carreto y Ruiz, 2012).

Los módulos trabajan con bases de datos, que contienen la información de los usuarios autorizados, los servicios educativos y los módulos educativos que requiere un usuario de acuerdo a la forma en que aprende y adquiere competencias.

Los Administradores de servicios educativos constan de varios puntos de acceso localizados en sitios estratégicos donde se brindará un servicio determinado, que puede constar del servicio de correo, acceso web, obtener información de un departamento, etc. que se dará automáticamente de acuerdo a los privilegios que le permita el administrador.

En la Figura 3 se muestra el modelo que se esta evaluando en un caso de prueba en la Escuela Superior de Cómputo del IPN; de un sistema de acceso a servicios educativos aplicados a la enseñanza AAVAE. El sistema cuenta con siete servicios: un blog móvil, un sistema de sesiones en línea para videoconferencia, un chat, foros, una biblioteca y servicio de archivos, cada servicio se puede encontrar distribuido en diferentes servidores.
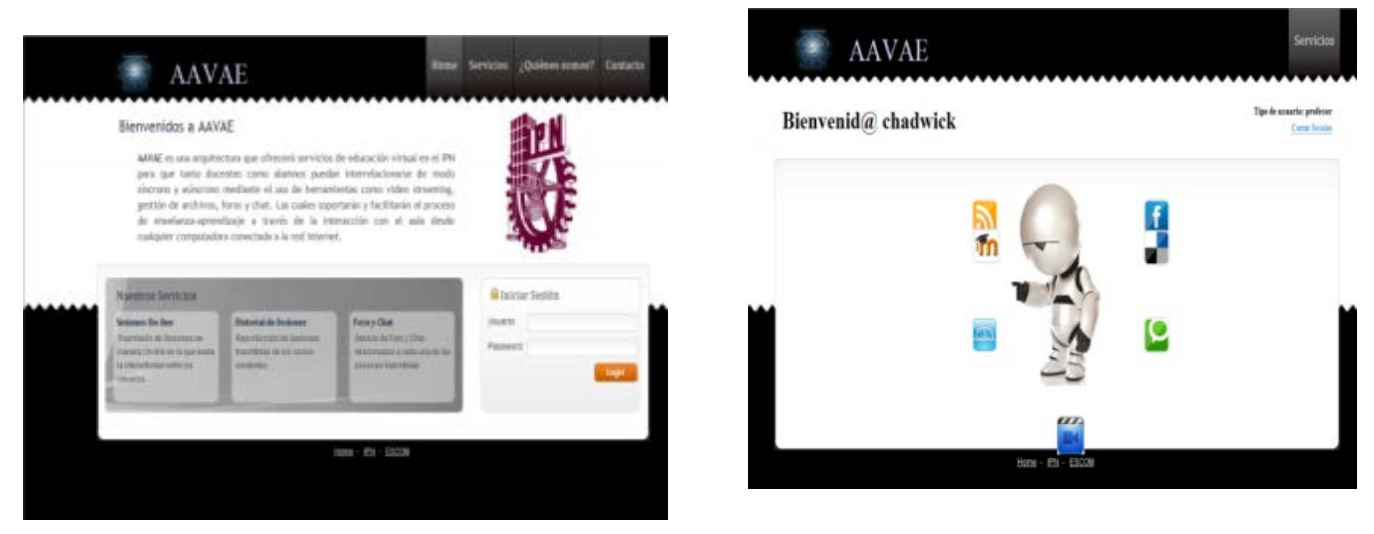

Figura 3. Sistema AAVAE.

Los servicios se desarrollaron en servicios Web y los clientes ligeros y respuestas trabajan con J2ME y XML en general. El cliente implementado en cada dispositivo trabaja con un cliente ligero pero no requiere de ningún instalable las pantallas de

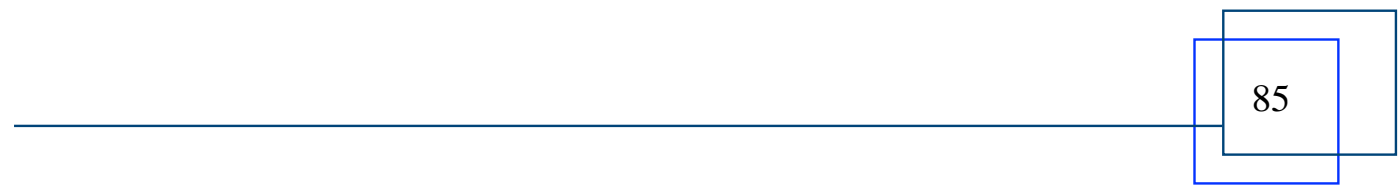




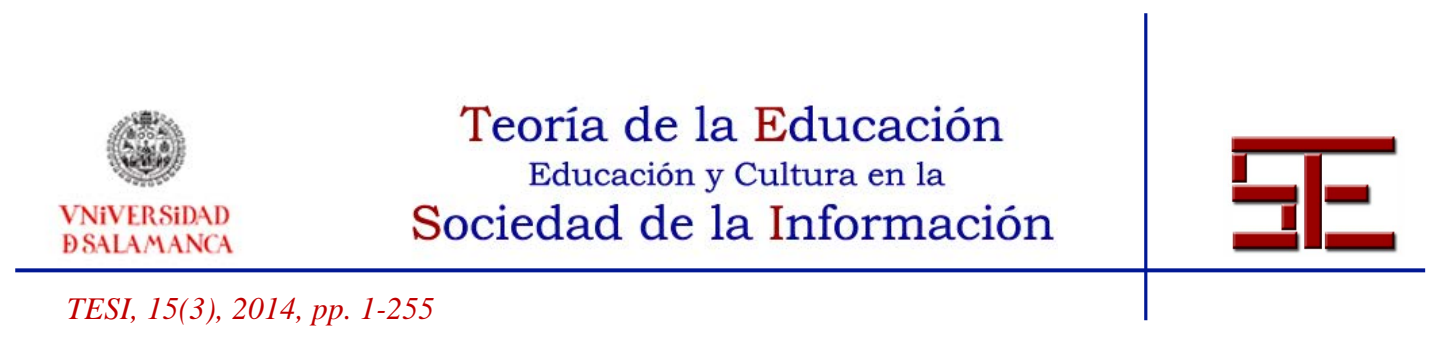

despliegue son estándar pero se tienen diferentes interfaces para adaptarse a cada tipo de cliente.

\section{IMPLEMENTACIÓN Y PRUEBAS}

La implementación del modelo en un caso de estudio se desarrolló en la Escuela Superior de Cómputo es una unidad académica del Instituto Politécnico Nacional que forma profesionales en sistemas computacionales a nivel licenciatura y posgrado. Actualmente cuenta con un número aproximado de 2.000 alumnos en la licenciatura y alrededor de 12 alumnos de posgrado. La oferta educativa en el área de posgrado es una Maestría en Ciencias en Sistemas Computacionales Móviles (Suárez et al., 2012).

Es en la unidad de aprendizaje "Desarrollo de Aplicaciones para Dispositivos Móviles" con la participación de 40 Alumnos de Licenciatura y en el laboratorio de la Maestría donde se implementó el caso de estudio del Modelo y donde se realizaron las pruebas sobre la implementación que se está presentando.

Las pruebas se realizaron para medir el grado de participación y de uso de información, recursos y servicios por parte de los alumnos de la unidad de aprendizaje "Desarrollo de Aplicaciones para Dispositivos Móviles" específicamente en el tema de "Desarrollo de Aplicaciones para Android". Con el uso de los sistemas y de la implementación del MAC se busca generar un mayor grado de desarrollo de competencias en la programación de Aplicaciones para dispositivos móviles.

\subsection{Estado inicial del caso de estudio}

Se realizó una evaluación del Grupo de la Unidad de Aprendizaje, una vez revisados los conceptos de desarrollo en Java para Aplicaciones sobre Sistema Operativo Android ver.2.0, el modelo educativo se basó en la impartición de cátedra y practicas guiadas y evaluadas durante 4 semanas, lo que generó un conjunto de prácticas y examen donde se evaluó el desempeño académico de los 40 alumnos.

Los resultados obtenidos en la evaluación se muestran en la siguiente tabla 


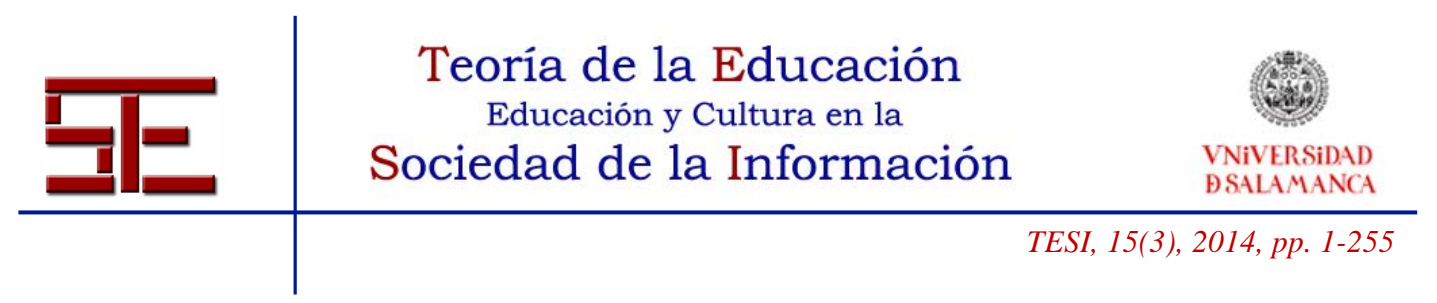

Tabla 1. Evaluación Inicial.

\begin{tabular}{|l|l|l|l|l|}
\hline $\begin{array}{l}\text { Alumnos } \\
\text { (40 Evaluados) }\end{array}$ & $\begin{array}{l}\text { Evaluación } \\
\text { Teórica } \\
\text { (Examen) }\end{array}$ & $\begin{array}{l}\text { Evaluación } \\
\text { Practica }\end{array}$ & \% Alumnos & $\begin{array}{l}\% \\
\text { Total }\end{array}$ \\
\hline $\begin{array}{l}100 \text { y 80\% de } \\
\text { aprovechamiento }\end{array}$ & 20 & 25 & 22.5 & $56.75 \%$ \\
\hline $\begin{array}{l}79 \text { y 60\% de } \\
\text { aprovechamiento }\end{array}$ & 10 & 10 & 10 & $25 \%$ \\
\hline $\begin{array}{l}\text { Menos de 60\% de } \\
\text { aprovechamiento }\end{array}$ & 10 & 5 & 7.5 & $18.25 \%$ \\
\hline
\end{tabular}

A partir de estos resultados se le generó una cuenta para ingresar al sistema de Apoyo académico a los alumnos para tener la información de la Unidad de Aprendizaje al alcance de sus requerimientos en el momento que lo necesitaran, por medio de cualquier dispositivo portátil con acceso a Internet.

La siguiente prueba de desarrollo sobre el mismo Tema de desarrollo en Java para Aplicaciones sobre Sistema Operativo Android, pero ahora para la versión 2.2, lo cual implica el cambio de librerías y lógica de programación de sensores y acelerómetros, el modelo educativo se basó en la asesoría por parte del Facilitador, el sistema se encargó por los medios de acceso al sistema de entregar a los alumnos la información necesaria de las librerías, de las bases técnicas de desarrollo en el momento que los alumnos la requerían y de dar seguimiento de las practicas propuestas durante 4 semanas.

\subsection{Pruebas realizadas}

Como se comentó anteriormente, se desarrollaron varias pruebas para comprobar el alcance que podría tener el Modelo implementado en sistema de Administración de Conocimiento por medio de entornos educativos. La prueba se desarrolló sobre el mismo Tema de desarrollo para Aplicaciones sobre Sistema Operativo Android para la versión 2.2, el sistema se encargó por los medios de acceso al sistema de entregar a los alumnos la información necesaria de las librerías, cada que los alumnos requerían información el sistema les descargaba periódicamente los documentos necesarios y daba acceso de acuerdo al avance del alumno a las practicas propuestas durante las 4 semanas de la prueba. 


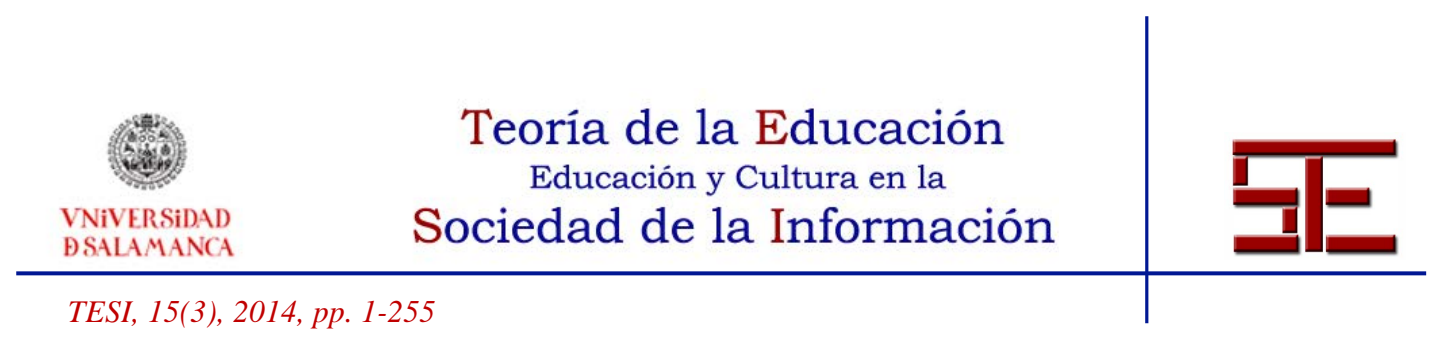

Para comprobar el alcance del modelo, se realizaron tres tipos de pruebas al caso de estudio, estás son:

- Acceso al sistema.

- Identificación de Perfiles para entrega de información.

- Acceso a los servicios y/o aplicaciones de Conocimiento.

\subsubsection{Prueba 1 - acceso al sistema:}

Esta prueba consistió en que los usuarios se conectaran al entorno educativo (sistema) implementado, recibieran la bienvenida al sistema, se autenticaran e hicieran uso de los servicios que se les ofrecieron de acuerdo a su perfil. La prueba se desarrolló durante un periodo de cuatro semanas y se llevó a cabo con los usuarios del curso y del laboratorio que asciende a aproximadamente 50 usuarios entre alumnos de licenciatura o maestría, profesores y algunos visitantes; sin embargo, la prueba se realizó con 40 de ellos. Cabe mencionar también que el acceso de estos no fue simultáneo, sino más bien intermitente, conforme se hacía uso del laboratorio. Esta prueba se realizó sobre gran variedad de dispositivos móviles de diferentes marcas, de 3ra generación (3G), los cuales incluyen con la tecnología WiFi como lo mencionamos en el capítulo anterior; tales como:

- Laptops: Dell, Acer, Apple, Compaq, Hp y Sony.

- Tablet PC: iPad y iPad2 de Apple y Galaxy de Samsung

- Teléfonos inteligentes: Blackberry, Nokia, Apple, Sony, Samsung.

De esta forma, se encuentra implícito que el modelo funciona también sobre diversas plataformas o sistemas operativos (Windows, Linux, Mac Os, Windows Mobile, Blackeberry OS, Android, Symbian) y diferentes navegadores de red (Opera mini, Explorer, Safari, etc.).

Los resultados obtenidos en esta prueba de acceso al sistema se presentan en la gráfica de la figura 5.7. La grafica indica que de una muestra de 40 usuarios el 97\% se conectó a la red de manera satisfactoria, mientras que el 3\% tuvo problemas de conexión, esto debido a un inconveniente que se tuvo con el punto de acceso inalámbrico y se tuvo que reiniciar este. 

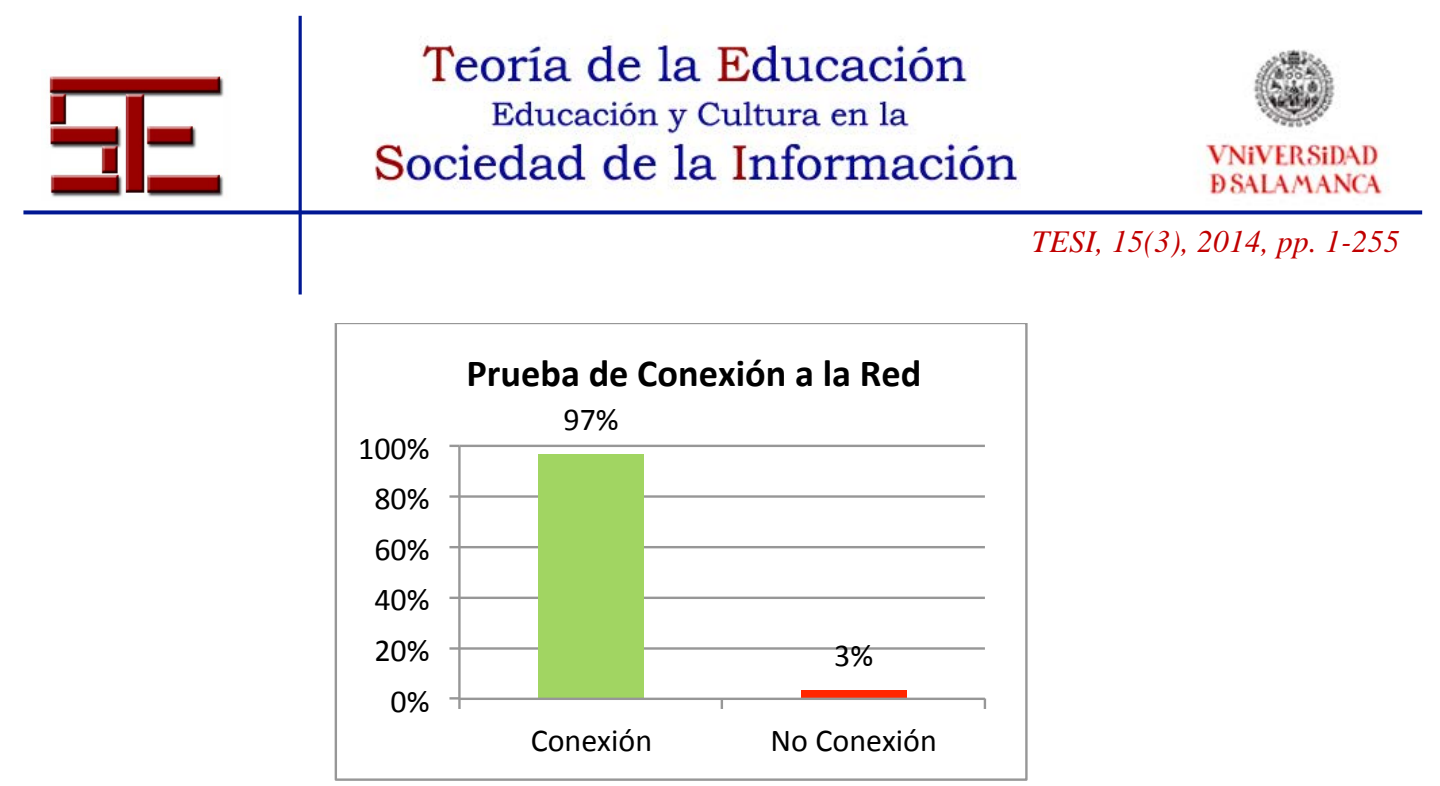

Figura 4. Gráfica de conexión y acceso al Sistema.

Para calcular la disponibilidad del acceso de red consideramos los 30 días (720 horas) que duró la prueba, así como los 30 minutos en que ese detuvo el servicio de red debido al problema que se suscitó en el AP, con ellos tenemos un tiempo de red disponible total de 719.5 horas. Utilizando la fórmula 5.1 obtenemos el porcentaje de disponibilidad.

$\%$ de disponibilidad $=\frac{\text { Tiempo de uso efectivo }}{\text { Tiempo de uso total }} \times 100$

Sustituyendo valores obtenemos:

- $\quad \%$ de disponibilidad $=(719.5 / 720) * 100$

- Disponibilidad del Dominio de Red $=99.93 \%$

Con el resultado anterior, se garantiza una buena disponibilidad del acceso al sistema.

\subsubsection{Prueba 2 -Identificación de perfiles para entrega de información}

La prueba consistió en que el usuario ingresara sus credenciales (nombre de usuario y contraseña) para poder autenticarse y obtener el acceso con sus servicios y recursos de acuerdo a su perfil. La figura 5.8 muestra los resultados obtenidos, en donde el 100\% de los usuarios no tuvieron problema alguno, es decir, se pudieron conectar y recibir su lista de servicios y/o aplicaciones disponibles de acuerdo a su perfil. Cabe aclarar que no se contabilizan los errores que surgieron cuando el usuario ingresó de manera incorrecta su propio usuario y contraseña.

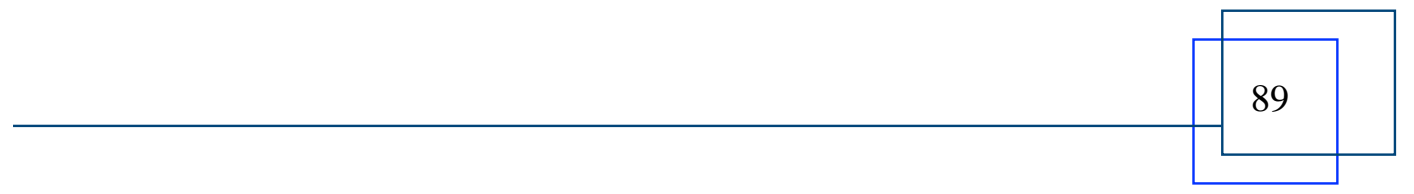



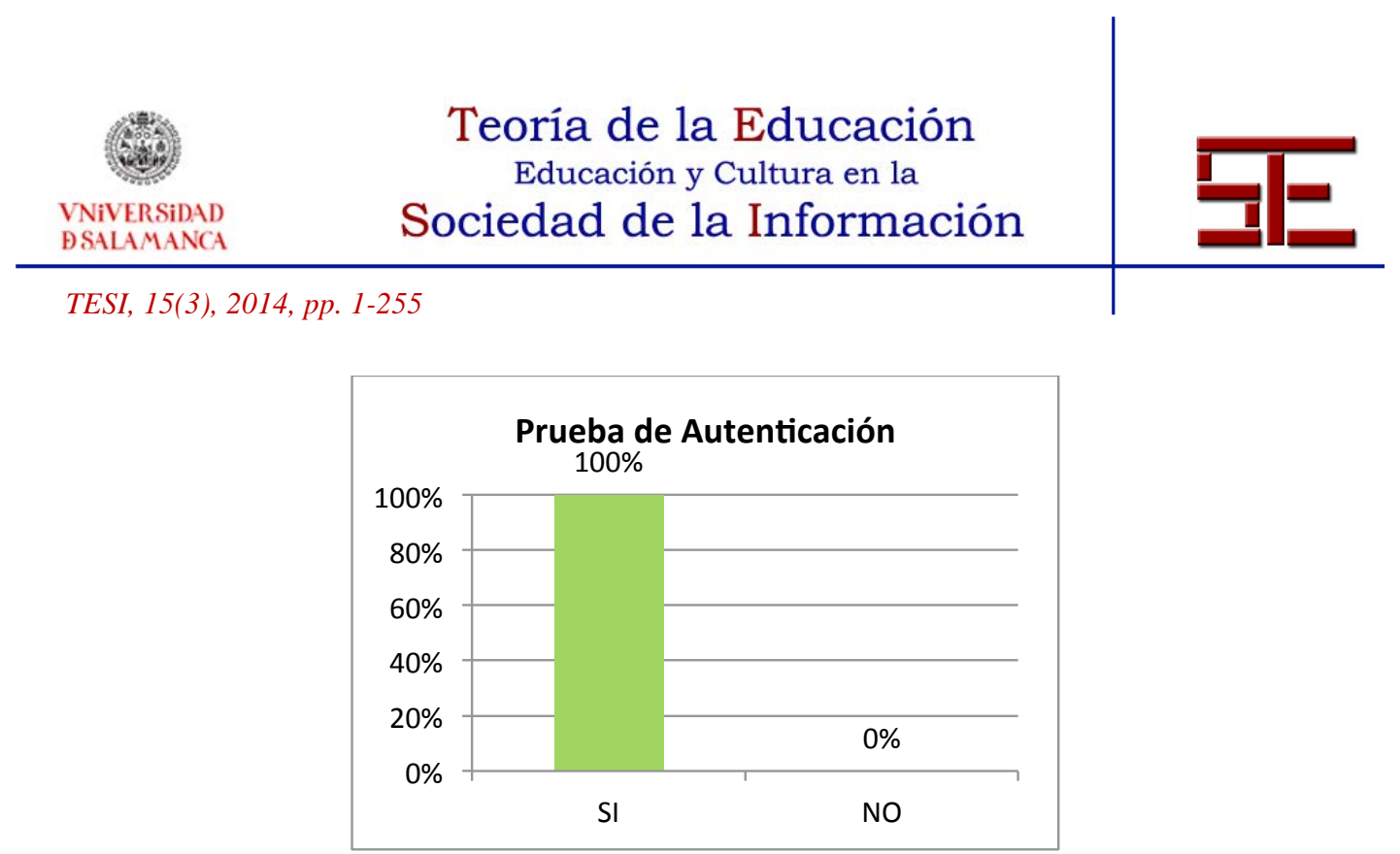

Figura 5. Gráfica de autenticación e identificación.

\subsubsection{Prueba 3 - Acceso a los servicios y/o aplicaciones de conocimiento}

La prueba de acceso a los servicios consistió en que el usuario accediera a una de las aplicaciones y/o servicios que tiene derecho una vez realizada la autenticación del usuario en el entorno educativo, esto con la finalidad de verificar la correcta disponibilidad de los servicios e Información ofrecidos, ya que esta es la base de generar el conocimiento necesario.

La figura 6 muestra las estadísticas del acceso de los servicios y/o aplicaciones que el usuario tiene derecho. El 95\% logró establecer de manera satisfactoria la conexión con los servicios, mientras que el $5 \%$ restante tuvo problemas debido a que el servidor que contenía el servicio no se encontraba disponible en ese momento.

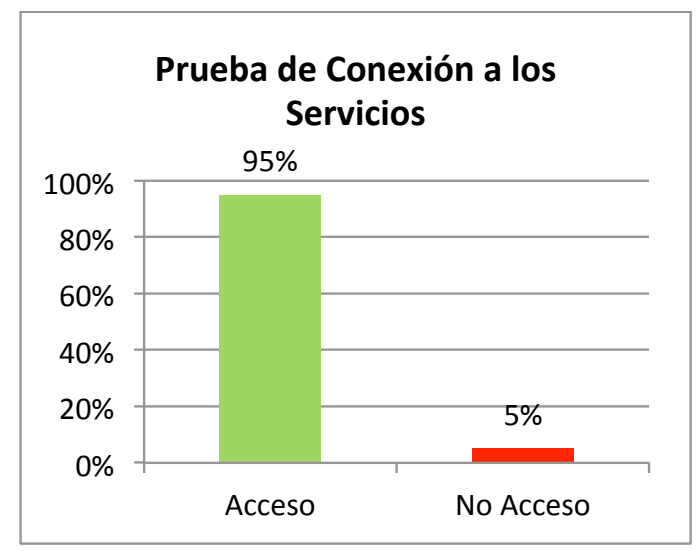

Figura 6. Gráfica de conexión a los servicios.

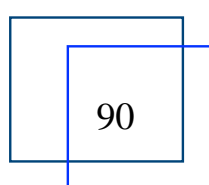




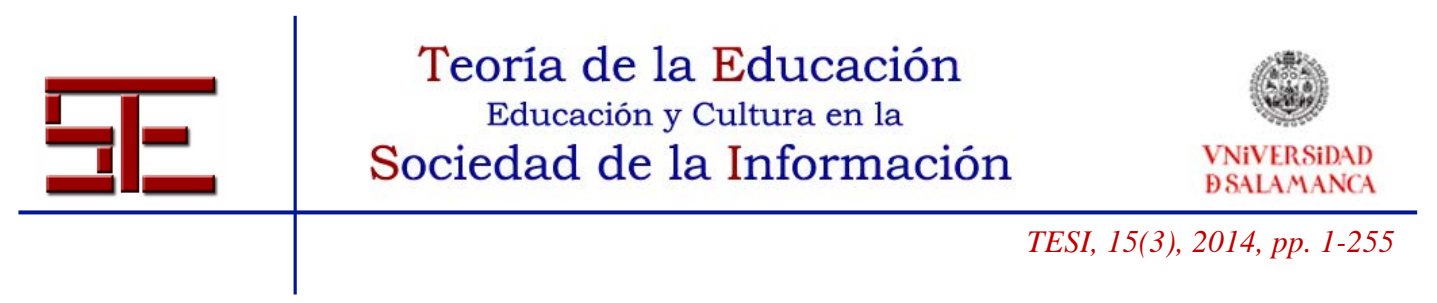

\section{ANÁLISIS CUALITATIVO DEL MAC}

Una vez realizadas las pruebas y definidas los resultados en las 4 semanas propuestas se realizó la evaluación de los Alumnos de la Unidad de Aprendizaje y se planteó el avance que tuvieron en varios aspectos, entre ellos:

- Conversión del Conocimiento Tácito a Explicito, poder compartir el conocimiento y comunicarlo y con esto generar y sobre todo evaluar competencias, se asume entonces que la conversión de conocimiento tácito en explícito es el mecanismo de transformación más rico en la creación de conocimiento.

- Uniformidad en la Información, obtener información interpretada, seleccionada, relacionada, organizada y, sobre todo, aplicada con pertinencia.

- Acceso Ilimitado, se obtiene la información precisa y útil en cualquier lugar y a cualquier hora (anytime, anywhere).

- Adaptable al cambio, acepta los cambios, es flexible y busca posibilidades y oportunidades de desarrollarse promoviendo nuevos planes y procesos.

Los resultados obtenidos en la segunda evaluación se muestran en la siguiente tabla

Tabla 2. Segunda Evaluación.

\begin{tabular}{|c|c|c|c|c|}
\hline $\begin{array}{l}\text { Alumnos } \\
\text { (40 Evaluados) }\end{array}$ & $\begin{array}{l}\text { Evaluación } \\
\text { Teórica } \\
\text { (Examen) }\end{array}$ & $\begin{array}{l}\text { Evaluación } \\
\text { Practica }\end{array}$ & $\%$ Alumnos & $\begin{array}{l}\% \\
\text { Total }\end{array}$ \\
\hline $\begin{array}{lcr}100 \mathrm{y} \quad 80 \% & \mathrm{de} \\
\text { aprovechamiento }\end{array}$ & 30 & 35 & 32.5 & $81.75 \%$ \\
\hline $\begin{array}{lcc}79 & \text { y } 60 \% & \text { de }\end{array}$ & 10 & 5 & 7.5 & $18.25 \%$ \\
\hline $\begin{array}{l}\text { Menos de } 60 \% \text { de } \\
\text { aprovechamiento }\end{array}$ & 0 & 0 & 0 & $0 \%$ \\
\hline
\end{tabular}

Los resultados obtenidos se compararon para definir ciertos aspectos sobre las definiciones anteriormente planteadas, tabla 3:

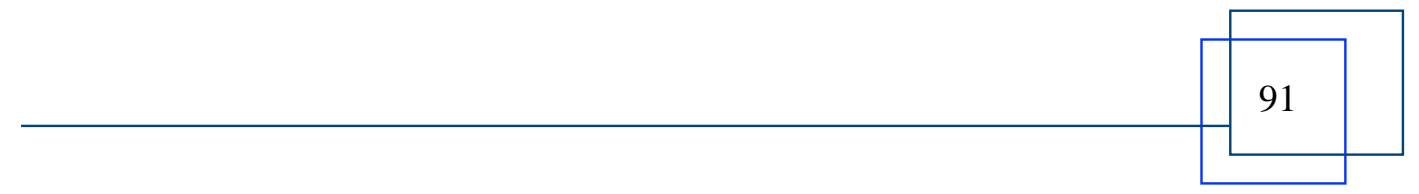




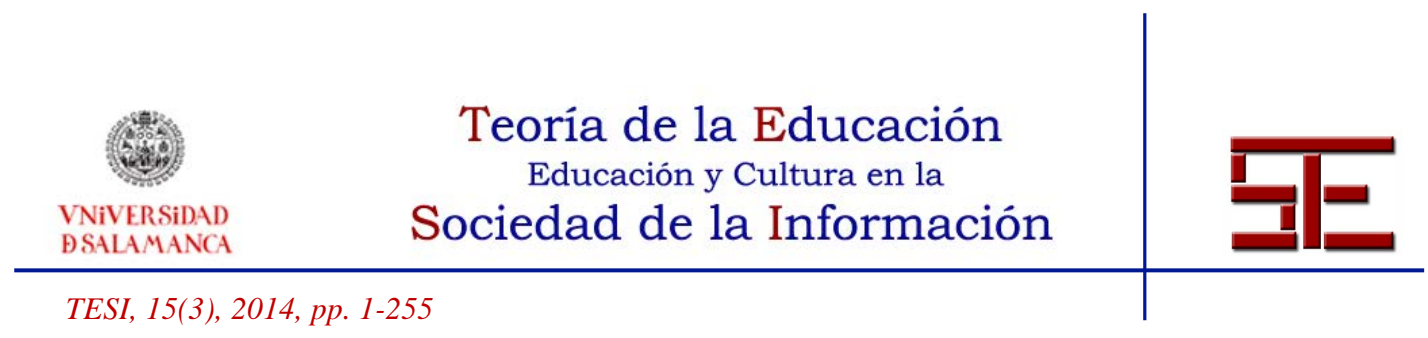

Tabla 3. Comparativa de Evaluaciones.

\begin{tabular}{|l|l|l|l|}
\hline $\begin{array}{l}\text { Alumnos } \\
\text { (40 Evaluados) }\end{array}$ & $\begin{array}{l}\text { Primera } \\
\text { Evaluación }\end{array}$ & $\begin{array}{l}\text { Segunda } \\
\text { Evaluación }\end{array}$ & Diferencia \\
\hline $\begin{array}{l}100 \text { y } 80 \% \text { de } \\
\text { Aprovechamiento }\end{array}$ & $56.75 \%$ & $81.75 \%$ & $+25 \%$ \\
\hline 79 y 60\% de Aprovechamiento & $25 \%$ & $18.25 \%$ & $-6.75 \%$ \\
\hline $\begin{array}{l}\text { Menos de 60\% de } \\
\text { Aprovechamiento }\end{array}$ & $18.25 \%$ & $0 \%$ & $-18.25 \%$ \\
\hline
\end{tabular}

El aumento de aprovechamiento en los alumnos se debe en términos generales por varios aspectos entre ellos y por los comentarios de los mismos alumnos en:

- Tener la información que requerían en el momento que requerían de acuerdo a las necesidades de desarrollo de sus actividades dentro de la Unidad de Aprendizaje. (Acceso Ilimitado).

- La información que llegaba a sus medios y dispositivos era la información precisa y relacionada, interpretada, seleccionada, etc. (Uniformidad en la Información).

- El sistema permitía compartir la experiencia y los resultados obtenidos, comunicarlos con sus compañeros y retroalimentar estas opiniones. (Conversión del Conocimiento Tácito a Explicito).

- Las prácticas avanzaban de acuerdo a los temas que se requería cubrir y esto generaba que se cambiaran las formas de modelado de las prácticas. (Adaptable al cambio).

\section{CONCLUSIONES Y TRABAJO A FUTURO}

La principal aportación del MAC propuesto es la de permitir actuar con más movilidad ahorrando tiempo y esfuerzo en el acceso a la información. El sistema por sí mismo es otra forma de ayudar a las personas a llevar a cabo sus funciones en cualquier área que se desarrollen, no solo porque facilita el trabajo, sino porque nos abre una puerta a la innovación y una contribución a la sociedad.

Una organización, y esto no excluye a las instituciones educativas, generalmente se rige por procesos que idealmente han sido verificados y certificados. Ello implica que la organización controla sus procesos para que los productos y servicios siempre se hagan 


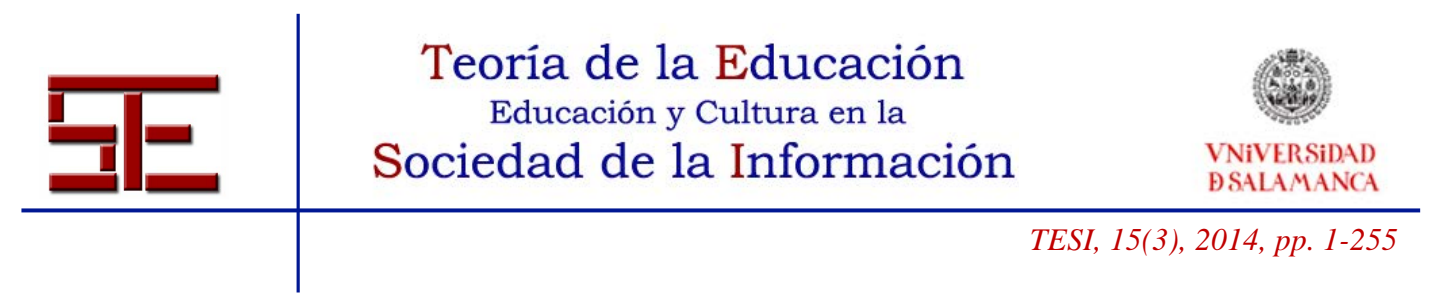

de la mejor forma. Las organizaciones e Instituciones corren el riesgo de quedarse estancadas con los procesos de globalización al ser superada por la competencia, pues no hay una estrategia de mejora continua donde se sistematice el conocimiento y se aprenda constantemente de los errores y desviaciones.

La tecnología constituye una parte decisiva del acceso externo, pero no por ello deja de ser una parte pequeña, sin embargo lo más importante es la capacidad intelectual el Conocimiento. Recabar información, sintetizarla, reflexionar sobre ella, discutirla, eso es lo esencial en la administración del conocimiento; la tecnología tiene que apoyar los cuatro tipos de actividad. El conocimiento jamás será definitivo. Se incuba y crece sin cesar.

Es importante realizar pruebas con múltiples usuarios para identificar el grado de conocimiento adquirido por medio del uso del modelo y la arquitectura de servicios educativos móviles propuesta.

\section{REFERENCIAS}

Attwell, G. (2007). Personal Learning Environments - the future of eLearning? In eLearning Papers, 2(1). Barcelona: elearningeuropa.info. Retrieved Retrieved December 18, 2008 Sitio web: <http://www.elearningeuropa.info/out/?doc_id=9758\&rsr_id=11561>.

Dutta S., y De Meyer A. (1997). Building Assets in Real Time and in Virtual Space. Denmark: Knowledge Management INSEAD.

Vygotsky, L. S. (2008). Pensamiento y Lenguaje. Madrid: Paidos,

Davenport T. H., Prusak L. (2001). Conocimiento en acción: Cómo las organizaciones manejan lo que saben. Buenos Aires: Pearson Educación.

Muñoz Duarte, M. A. (2003). Cómputo colaborativo consciente del contexto. Tesis de Maestría, México, CICESE.

Akyildiz, I. A. (2012). A Survey on Sensor Network. EUA. IEEE Communications Magazine.

Humanante Ramos, P., García-Peñalvo, F. J., Conde González, M. Á. (2013). Entornos Personales de Aprendizaje y Aulas Virtuales: Una Experiencia con Estudiantes Universitarios. IEEE VAEP-RITA. 1(4): 211-217.

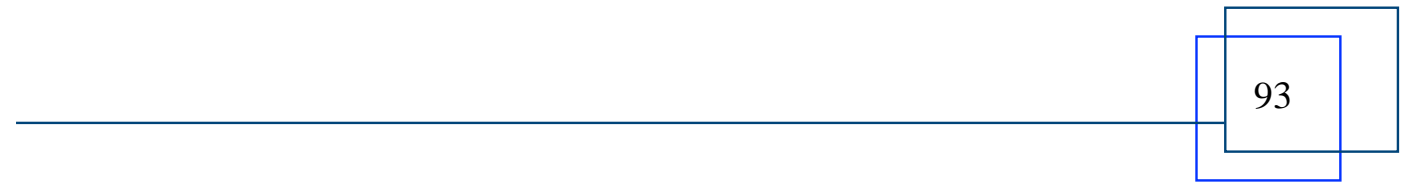




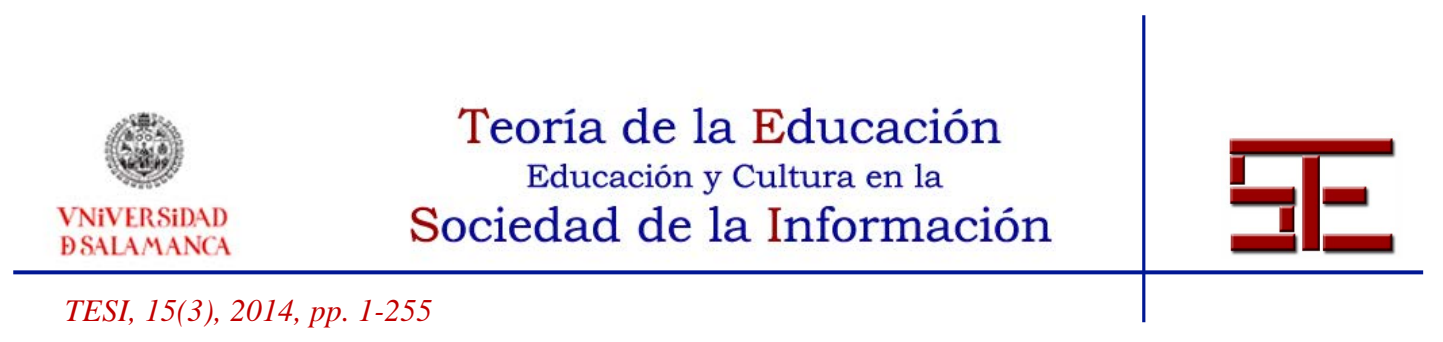

Adell, J. \& Castañeda, L. (2010). Los Entornos Personales de Aprendizaje (PLEs): una nueva manera de entender el aprendizaje. In R. Roig Vila \& M. Fiorucci (Eds.), Claves para la investigación en innovación y calidad educativas. La integración de las Tecnologías de la Información y la Comunicación y la Interculturalidad en las aulas. Alcoy: Marfil.

Castañeda, L. \& Adell, J. (2013). PLE: Una Perspectiva Tecnológica. In L. Castañeda \& J. Adell., Entornos Personales de Aprendizaje: claves para el ecosistema educativo en red (pp. 71-84). Alcoy: Marfil.

García-Peñalvo, F. J., Conde, M. Á., Pozo, A. del (2013). A Mobile Personal Learning Environment Approach. In Virtual, Augmented and Mixed Reality. Systems and Applications. 5th International Conference, VAMR 2013 Held as Part of HCI International 2013 Las Vegas, NV, USA, July 21-26, 2013. Proceedings, Part II (Las Vegas, Nevada, USA, 21-26 July 2013) (pp. 132-141).

Fidalgo Blanco, Á., Lerís, D., Sein-Echaluce, M. ${ }^{a}$ L., García-Peñalvo, F. J. Indicadores para el seguimiento y evaluación de la competencia de trabajo en equipo a través del método CTMTC. En Á. Fidalgo Blanco, $\mathrm{M}^{\mathrm{a}}$ L. Sein-Echaluce Lacleta (Eds.). Actas del II Congreso Internacional sobre Aprendizaje, Innovación y Competitividad, CINAIC 2013 (Madrid, 6-8 de noviembre de 2013). (pp. 280-285). Madrid, España: Fundación General de la Universidad Politécnica de Madrid.

Conde, M. Á., García-Peñalvo, F. J. "Entornos Personales de Aprendizaje móviles y su aplicación en la enseñanza de Ingeniería del Software”. En Á. Fidalgo Blanco, M. ${ }^{a}$ L. Sein-Echaluce Lacleta (Eds.). Actas del II Congreso Internacional sobre Aprendizaje, Innovación y Competitividad, CINAIC 2013 (Madrid, 6-8 de noviembre de 2013). (pp. 691-696). Madrid, España: Fundación General de la Universidad Politécnica de Madrid.

Conde González, M. Á., García-Peñalvo, F. J. (2013). Experiencia de Aplicación de un Entorno Personalizado de Aprendizaje Móvil a una Asignatura de Ingeniería Informática". IEEE VAEP-RITA. 1(4): 193-200.

Sánchez Prieto, J. C., Olmos Migueláñez, S., García-Peñalvo, F. J. (2014). Understanding mobile learning: devices, pedagogical implications and research lines. Teoría de la Educación: Educación y Cultura en la Sociedad de la Información, 15(1):20-42.

<http://campus.usal.es/ revistas_trabajo/index.php/revistatesi/article/view/11563/11983 $>$. 


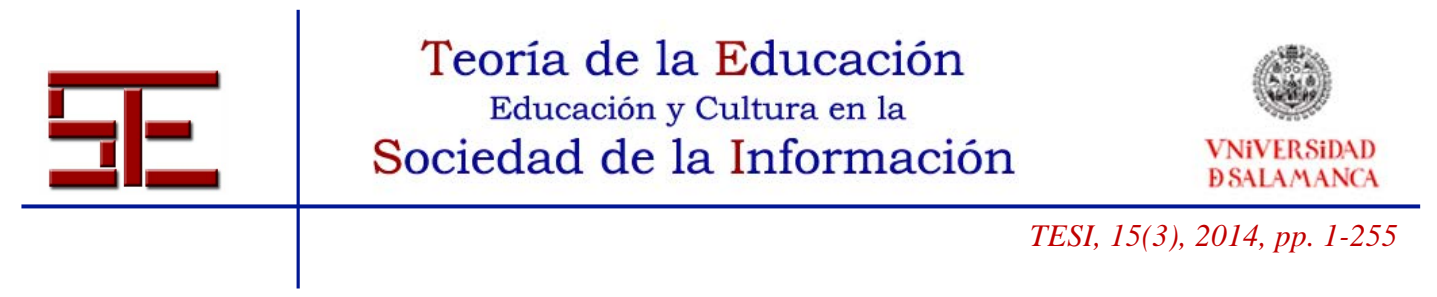

Suárez, S., Carreto, C., y Ruiz F. (2012). Modelo de Administración del Conocimiento aplicado al proceso de Enseñanza-Aprendizaje por medio de Dispositivos Móviles. Tesis de Maestría, SEPI-ESCOM, Instituto Politécnico Nacional. México.

Agradecimientos. Los autores agradecen las facilidades otorgada para la realización de este trabajo a IPN, CONACYT, UPICSA, ESCOM, COFFA, SIP-IPN. 